\title{
Adaptive Event-Triggered Synchronization of Networked Neural Networks with Time-Varying Delay Subject to Actuator Saturation
}

\author{
Yao Xu $\mathbb{D}^{1},{ }^{1}$ Renren Wang $\mathbb{D}^{1},{ }^{1}$ Hongqian Lu $\mathbb{D},{ }^{1}$ Xingxing Song $\mathbb{D},{ }^{1}$ Yahan Deng $\mathbb{D},{ }^{1}$ \\ and Wuneng Zhou ${ }^{2}$ \\ ${ }^{1}$ School of Electrical Engineering and Automation, Qilu University of Technology (Shandong Academy of Sciences), \\ Jinan 250353, China \\ ${ }^{2}$ College of Information Science and Technology, Donghua University, Shanghai 201620, China
}

Correspondence should be addressed to Renren Wang; wrr@qlu.edu.cn

Received 8 March 2021; Revised 10 June 2021; Accepted 28 June 2021; Published 7 July 2021

Academic Editor: Thach Ngoc Dinh

Copyright $(0) 2021$ Yao Xu et al. This is an open access article distributed under the Creative Commons Attribution License, which permits unrestricted use, distribution, and reproduction in any medium, provided the original work is properly cited.

This paper discusses the adaptive event-triggered synchronization problem of a class of neural networks (NNs) with time-varying delay and actuator saturation. First, in view of the limited communication channel capacity of the network system and unnecessary data transmission in the NCSs, an adaptive event-triggered scheme (AETS) is introduced to reduce the network load and improve network utilization. Second, under the AETS, the synchronization error model of the delayed master-slave synchronization system is constructed with actuator saturation. Third, based on Lyapunov-Krasovskii functional (LKF), a new sufficient criterion to guarantee the asymptotic stability of the synchronization error system is derived. Moreover, by solving the stability criterion expressed in the form of a set of linear matrix inequalities (LMIs), some necessary parameters of the system are obtained. At last, two examples are expressed to demonstrate the feasibility of this method.

\section{Introduction}

NNs represent a computing type similar to that of the brain [1]. Now, they have been widely applied in many fields, such as secure communications, combinatorial optimization, pattern recognition, associative memories, and complex system control [2-8]. At the same time, they are also the key feature of fitting nonlinear functions and recognizing patterns, which makes NNs can be specifically applied to aerospace, electronics, finance, automobile, banking, telecommunications, national defense, manufacturing, robotics, and transportation industries [1]. As we all know, the development of many systems is related not only to the current time state but also to the state before and after the current time point. In many practical systems, the time delay is inevitable, which is usually the main reason for the instability of practical systems [9]. Because of network congestion and limited signal transmission speed between neurons, the time delay is common in NNs. Therefore, the delay is introduced into NNs, and as a kind of complex nonlinear system, delayed NNs (DNNs) can exhibit complicated dynamic behaviors effectively and even chaotic phenomena, so it has received considerable attention.

Synchronization refers to the coordination of events occurring in a system, and the phenomenon of consistency and unification appears in time. That is to say, by adopting appropriate control strategies, the trajectory of the slave system under different initial conditions can be consistent with that of the master system after a certain time. Synchronization as a self-organizing form is one of the fundamental phenomena in the universe, which can be used in many practical problems, such as wireless sensor networks, chaotic circuits, and two-way teleoperation systems. The related synchronization problems have gradually become the focus of researchers. Many control methods for keeping the synchronization of NNs have also been proposed, including adaptive control [10-12], sampled-data control $[8,13,14]$, intermittent control [15], sliding mode control [16], and impulsive control. By using an intermittent control method [15], focused on the exponential synchronization 
and problem of L2-gain analysis of delayed chaotic NNs with actuator saturation, and by solving LMIs, the intermittent linear state feedback controller was obtained. In [17], considering the actuator saturation, sampled-data synchronization of chaotic NNs was discussed; the condition of keeping local synchronization of master-slave NNs was derived; and the relevant sampling data controllers can be obtained by employing LMI.

Considering the limited range of amplitude signals generated by actuators in practical systems, actuator saturation is a common phenomenon and widely considered in control systems [18]. Because actuator saturation is inevitable, it may lead to the deterioration of system performance, and even worse, it may make the closed-loop system unstable, so the research on actuator saturation is of great significance, and a lot of work has been done [1, 9, 18-23]. For instance, in [1], using the quantized measurement method, the synchronization problem of DNNs was discussed with actuator saturation. In [19], starting from the concept of master-slave synchronization, the author discussed the synchronization of drive-response systems with input delay and saturation. In [20], on the premise of fully considering the influence of asynchronous preconditions caused by event-triggered sampling, the event-triggered master-slave synchronization problem of delayed T-S fuzzy neural networked systems was discussed. Considering actuator saturation, the impulsive synchronization problem of chaotic DNNs was discussed in [22]. In [23], the synchronization of delayed dynamic networks was discussed with actuator saturation.

Compared with traditional control systems, NCSs have the advantages of low cost, less wiring, easy diagnosis, maintenance [24], etc. Based on this, this paper brings a synchronization system into the framework of NCSs. As we know, because of the limited communication channel capacity of the network system and bandwidth [25-27], delay and congestion often occur in the network channels. Therefore, the basic problems that need to be solved in NCSs are: whether there has enough communication bandwidth to feed the information back to the controller and then send the control command to actuators and equipment [28] and whether the communication bandwidth can be used efficiently when we consider the NCSs. Usually, in order to realize and analyze the system easily, many literature works use the periodic trigger control method to model and analyze the system. With this trigger method, a fixed and suitable sampling interval is selected to ensure the expected performance under the conditions of external interference, uncertainty, and time delay [29, 30]. However, the simple periodic trigger control method will obviously waste the limited communication bandwidth.

On the other hand, stability analysis is one of the basic problems of time-delay systems. One of its main objectives is to determine and maximize the maximum time delay upper bound for keeping the system stable. As we all know, the LKF method is a proven powerful tool to study the stability of time-delay systems. By constructing an appropriate LKF and using an appropriate integration processing technique to estimate the integral term in the derivative of LKF, the judgment condition of system stability is finally obtained. What is worth mentioning here is that some scholars have found that the time delay in the system may lead to the improvement rather than a deterioration of the system performance when using the looped-function [31] to study the stability of the aperiodic sampled-data control system with time delay [32], which deserves further study. For the estimation of integral term, appropriate integral inequalities can be chosen flexibly. The popular methods are the freeweight matrix method, the second-order Bessel-Legendre inequality [33], the Jacobi-Bessel inequality [34], and the quadratic function negative-determination lemma [35].

Based on the above analysis, this note investigates the synchronization error problem of a class of master-slave DNNs under the AETS [10-12]. The synchronization error model is built on the framework of NCSs, where both actuator saturation and network-induced delay are considered. Specifically, it can be divided into the following points. First, we transformed the synchronization problem of masterslave DNNs into the stability problem of the synchronization error system, and the actuator saturation and network-induced delay were considered simultaneously. Second, aiming at the limited communication channel capacity of the network system, the AETS is introduced to replace the traditional periodic sampling, thus reducing the network load and saving network resources. Finally, by using Lyapunov stability theory, a new sufficient criterion expressed in the form of LMIs is given to guarantee the stability of the synchronization error system.

Notations: $\mathbb{R}^{n}$ means $n$-dimensional Euclidean space, $\mathbb{R}^{m \times n}$ means $m \times n$ real matrices, $I$ denotes the identity matrix, block diagonal matrix is represented by $\operatorname{diag}\{\ldots\}$, and $*$ is used to denote symmetry terms in a matrix.

\section{Problem Formulation and Preliminaries}

In this section, we first establish a synchronous error model with actuator saturation based on AETS. At the same time, some lemmas, assumptions, and definitions are given to facilitate subsequent understanding.

2.1. The Master-Slave DNNs and AETS. Consider the following master-slave DNNs represented by $\mathscr{M}-\mathcal{S}$ :

$$
\begin{aligned}
\mathscr{M}: \dot{x}(t)= & -A x(t)+W_{1} f(x(t))+W_{2} f(x(t-d(t))), \\
\mathcal{S}: \dot{y}(t)= & -A y(t)+W_{1} f(y(t))+W_{2} f(y(t-d(t))) \\
& +\operatorname{sat}(u(t)),
\end{aligned}
$$

where $A, W_{1}$ and $W_{2}$ are given system matrices; $x(t), y(t) \in \mathbb{R}^{n}$ are the state vectors; $u(t) \in \mathbb{R}^{m}$ means the control input; the interval time-varying delay is $d(t)$, which satisfies $0 \leq d(t) \leq d_{M}$; and $\operatorname{sat}(u(t))$ means the saturation nonlinearity function of the control input $u(t)$ denoted by sat $(\cdot): \mathbb{R}^{m} \rightarrow \mathbb{R}^{m}$, which is recorded as follows:

$$
\operatorname{sat}(u):=\left[\operatorname{sat}\left(u_{1}\right), \operatorname{sat}\left(u_{2}\right), \ldots, \operatorname{sat}\left(u_{m}\right)\right]^{T},
$$

where we have $\operatorname{sat}\left(u_{i}\right)=\operatorname{sgn}\left(u_{i}\right) \min \left\{\mu_{i},\left|u_{i}\right|\right\}$, and we can write it as follows: 


$$
\operatorname{sat}\left(u_{i}\right)= \begin{cases}\mu_{i}, & u_{i}>\mu_{i}, \\ u_{i}, & -\mu_{i} \leq u_{i} \leq \mu_{i}, i=1,2, \ldots, m, \\ -\mu_{i}, & u_{i}<-\mu_{i} .\end{cases}
$$

$f(\cdot) \in \mathbb{R}^{n}$ denotes the neuron activation function, and we assume: $f(\cdot)$ is continuous and bounded; then for any $\alpha_{1}, \alpha_{2} \in \mathbb{R}\left(\alpha_{1} \neq \alpha_{2}\right)$ and known positive scalars $\varrho_{i}(i=1,2, \ldots, n)$, we have

$$
0 \leq \frac{f_{i}\left(\alpha_{1}\right)-f_{i}\left(\alpha_{2}\right)}{\alpha_{1}-\alpha_{2}} \leq \varrho_{i} .
$$

Next, we will give two common assumptions in NCSs research.
Assumption 1. There is always a time delay in signal transmission, suppose that the network-induced delay $\tau_{k} \in(0, \bar{\tau}), k \in\{0,1,2, \ldots$,$\} , and \bar{\tau}>0$ is the upper bound of $\tau_{k}$. So if the data is released at $t_{k} h$, at the time $t_{k} h+\tau_{k}$, it will arrive at the actuator.

Assumption 2. Assume that the sampling sequence is $\{0, h, 2 h, \ldots, n h\}, t_{k} h$ and $t_{k+1} h$ are the current release time and the next release time respectively. Moreover, $t_{k+1} h=t_{k} h+n h$, where $n h$ means the release interval of the transmitted data.

Then, considering the delay in the process of network transmission and combined with the structure of the eventtriggered synchronization system shown in Figure 1, we can construct a delayed network model for the synchronization system. Define that

$$
\begin{aligned}
m_{k} & =\min \left\{\lambda \mid t_{k} h+\tau_{k}+\lambda h \geq t_{k+1} h+\tau_{k+1}, \quad \lambda=0,1,2, \ldots,\right\}, \\
\Pi_{\lambda}^{\prime} & =\left[t_{k} h+\tau_{k}+(\lambda-1) h, t_{k} h+\tau_{k}+\lambda h\right), \quad \lambda=0,1,2, \ldots, m_{k}-1, \\
\Pi_{m_{k}}^{\prime} & =\left[t_{k} h+\left(m_{k}-1\right) h+\tau_{k}, t_{k+1} h+\tau_{k}\right),
\end{aligned}
$$

and we can rewrite the interval $\left[t_{k} h+\tau_{k}, t_{k+1} h+\tau_{k+1}\right)$ as follows:

$$
\left[t_{k} h+\tau_{k}, t_{k+1} h+\tau_{k+1}\right)=\bigcup_{\lambda=1}^{m_{k}} \Pi_{\lambda}^{\prime}
$$

further defining that

$$
\begin{gathered}
h(t)= \begin{cases}t-t_{k} h, & t \in \Pi_{1}^{\prime} \\
t-t_{k} h-h, & t \in \Pi_{2}^{\prime} \\
\vdots & \vdots \\
t-t_{k} h-\left(m_{k}-1\right) h, & t \in \Pi_{m_{k}}^{\prime}\end{cases} \\
e_{k}(t)= \begin{cases}0, & t \in \Pi_{1}^{\prime}, \\
\eta\left(t_{k} h\right)-\eta\left(t_{k} h+h\right), & t \in \Pi_{2}^{\prime}, \\
\vdots & \vdots \\
\eta\left(t_{k} h\right)-\eta\left(t_{k} h+\left(m_{k}-1\right) h\right), & t \in \Pi_{m_{k}}{ }^{\prime},\end{cases}
\end{gathered}
$$

where $\quad 0<\tau_{k} \leq h(t) \leq \bar{\tau}+h$. Let $\quad h_{M}=\bar{\tau}+h$, then $0<\tau_{k} \leq h(t) \leq h_{M}$.

In view of the limited communication channel capacity of the NCSs and reduce unnecessary data transmission, the AETS is introduced shown as follows:

$$
e_{k}^{T}(t) \Omega e_{k}(t) \leq \sigma(t) \eta^{T}(t-h(t)) \Omega \eta(t-h(t)),
$$

and $\sigma(t)$ satisfies

$$
\dot{\sigma}(t)=\frac{1}{\sigma(t)}\left(\frac{1}{\sigma(t)}-\vartheta\right) e_{k}^{T}(t) \Omega e_{k}(t),
$$

where $\sigma(t) \in(0,1), \vartheta>0$, and $\Omega>0$ are the parameters of AETS to be determined.
Remark 1. From AETS (9), we know that the traditional time-triggered scheme is only a special case when $\sigma=0$, so the AETS adopted here has higher flexibility and wider application range.

According to formulae (7), and (8), in this paper, the controller can be designed as follows:

$$
u(t)=K \eta\left(t_{k} h\right)=K\left(\eta(t-h(t))+e_{k}(t)\right),
$$

where $K$ is the controller gain matrix.

2.2. The Synchronization Error System and Lemmas. By defining $\eta(t)=y(t)-x(t)$, the master-slave DNNs synchronization error can be obtained as follows:

$$
\dot{\eta}(t)=-A \eta(t)+W_{1} g(\eta(t))+W_{2} g(\eta(t-d(t)))+\operatorname{sat}(u(t)),
$$

where $g(\eta(t))=f(y(t))-f(x(t))$, and we can know it from equation (4) that for any $i=1,2, \ldots, n$

$$
0 \leq \frac{f_{i}\left(y_{i}(t)\right)-f_{i}\left(x_{i}(t)\right)}{y_{i}(t)-x_{i}(t)}=\frac{g_{i}\left(\eta_{i}(t)\right)}{\eta_{i}(t)} \leq \varrho_{i} .
$$

To process the nonlinear part sat $(u(t))$, Lemma 1 is used here.

Lemma 1 (see [22]). For $\psi(u(t))=\left[\psi_{1}(u(t)), \quad \psi_{2}(u\right.$ $\left.(t)), \ldots, \psi_{m}(u(t))\right]^{T} \in \mathbb{R}^{m}$, the saturation function sat $(u(t))$ can be expressed as follows:

$$
\operatorname{sat}(u(t))=u(t)-\psi(u(t))
$$

where $u(t)$ is a linear part and $\psi(u(t))$ is a nonlinear part, and there exists a scalar $\varepsilon \in(0,1)$, which makes the inequality 


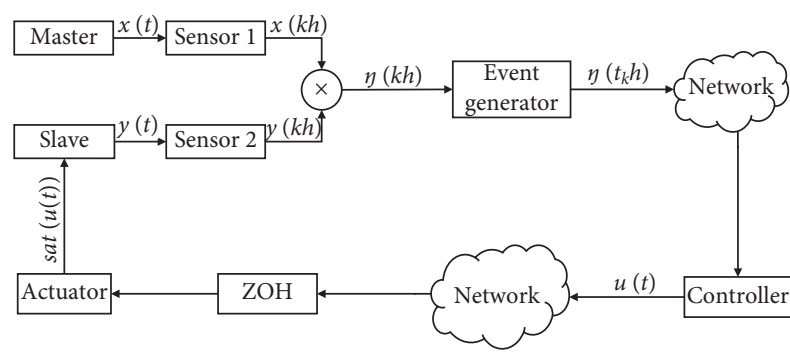

FIgURE 1: The block diagram of the event-triggered synchronization system.

$$
\psi(u(t))^{T} \psi(u(t)) \leq \varepsilon u(t)^{T} u(t)
$$

hold.

Based on Lemma 1 and equation (11), we can rewrite sat $(u(t))$ as follows:

$$
\operatorname{sat}(u(t))=K\left(\eta(t-h(t))+e_{k}(t)\right)-\psi(u(t)) .
$$

Using equation (16), replace sat $(u(t))$ in equation (12); then we can rewrite it as follows:

$$
\begin{aligned}
\dot{\eta}(t)= & -A \eta(t)+W_{1} g(\eta(t))+W_{2} g(\eta(t-d(t))) \\
& +K\left(\eta(t-h(t))+e_{k}(t)\right)-\psi(u(t)) .
\end{aligned}
$$

Next, we will give some other main lemmas.

Lemma 2. Consider $0 \leq h(t) \leq h_{M}$, for any matrices $\mathscr{Z} \in \mathbb{R}^{n \times n}$ and $\mathbb{Q} \in \mathbb{R}^{n \times n}$ that satisfy $\left[\begin{array}{cc}\mathscr{Z} & \mathbb{Q} \\ \mathscr{Q}^{T} & \mathscr{Z}\end{array}\right] \geq 0$, then the following inequality holds:

$$
\begin{aligned}
& -h_{M} \int_{t-h_{M}}^{t} \dot{x}^{T}(s) \mathscr{Z} \dot{x}(s) \mathrm{d} s, \\
\leq & {\left[\begin{array}{c}
x(t) \\
x(t-h(t)) \\
x\left(t-h_{M}\right)
\end{array}\right]^{T}\left[\begin{array}{ccc}
-\mathscr{E} & * & * \\
\mathscr{E}^{T}-\mathscr{Q}^{T} & -2 \mathscr{Z}+\mathscr{Q}+Q^{T} & * \\
\mathscr{Q}^{T} & \mathscr{E}^{T}-Q^{T} & -\mathscr{Z}
\end{array}\right]\left[\begin{array}{c}
x(t) \\
x(t-h(t)) \\
x\left(t-h_{M}\right)
\end{array}\right] . }
\end{aligned}
$$

Lemma 3 (see [36]). Suppose that $\Phi_{1}, \Phi_{2}$, and $\Phi_{3}$ are appropriate matrices and $0 \leq d_{m} \leq d(t) \leq d_{M}$, then

$$
\left(d(t)-d_{m}\right) \Phi_{1}+\left(d_{M}-d(t)\right) \Phi_{2}+\Phi_{3}<0,
$$

if and only if

$$
\begin{aligned}
& \left(d_{M}-d_{m}\right) \Phi_{1}+\Phi_{3}<0 \\
& \left(d_{M}-d_{m}\right) \Phi_{2}+\Phi_{3}<0
\end{aligned}
$$

hold.

Lemma 4 (see [37]). For any scalar $\rho$ and matrices $M>0$ and $N=N^{T}$, the inequality

$$
-N M^{-1} N \leq \rho^{2} M-2 \rho N,
$$

\section{Main Results}

In this section, we will give the asymptotic stability criterion of the synchronization error system (17) using LKF under the AETS in Theorem 1. Then considering the nonlinear terms in Theorem 1, we will give its explicit expression in Theorem 2 .

Theorem 1. For given parameters $h_{M}>0, d_{M}>0$ and positive scalars $\lambda, \alpha, \beta$, and $\varepsilon, \sigma(0) \in(0,1)$, under AETS (9), synchronization error system (17) subject to actuator saturation is asymptotically stable, if there exist appropriate matrices $P>0, Q_{i}>0, R_{i}>0, M_{i}, N_{i}(i=1,2)$, and $U$, which satisfy the following matrix inequalities:

$$
\begin{gathered}
\Psi_{i}=\left[\begin{array}{cccc}
\Xi & * & * & * \\
\Lambda & -\Psi_{22}^{-1} & * & * \\
\Gamma & 0 & -I & * \\
\Pi_{i} & 0 & 0 & -d_{M} R_{2}
\end{array}\right]<0 \quad(i=1,2), \\
{\left[\begin{array}{cc}
R_{1} & * \\
U & R_{1}
\end{array}\right]>0,}
\end{gathered}
$$

where

$$
\Xi=\left[\begin{array}{ccccccccc}
\Xi_{11} & * & * & * & * & * & * & * & * \\
\Xi_{21} & \Xi_{22} & * & * & * & * & * & * & * \\
U & R_{1}-U & \Xi_{33} & * & * & * & * & * & * \\
\Xi_{41} & 0 & 0 & \Xi_{44} & * & * & * & * & * \\
0 & 0 & 0 & -N_{1}^{T}+N_{2} & \Xi_{55} & * & * & * & * \\
\Xi_{61} & 0 & 0 & 0 & 0 & -2 \alpha I & * & * & * \\
W_{2}^{T} P & 0 & 0 & \beta \Sigma & 0 & 0 & -2 \beta I & * & * \\
-P & 0 & 0 & 0 & 0 & 0 & 0 & -\lambda I & * \\
K^{T} P & 0 & 0 & 0 & 0 & 0 & 0 & 0 & -\Omega
\end{array}\right],
$$$$
\Xi_{11}=-R_{1}+Q_{1}+Q_{2}-P A-A^{T} P+M_{1}+M_{1}^{T} \text {, }
$$$$
\Xi_{21}=K^{T} P+R_{1}-U \text {, }
$$$$
\Xi_{22}=-2 R_{1}+U+U^{T}+\sigma(t) \Omega \text {, }
$$$$
\Xi_{33}=-R_{1}-Q_{1} \text {, }
$$$$
\Xi_{41}=-M_{1}^{T}+M_{2} \text {, }
$$$$
\Xi_{44}=-M_{2}-M_{2}^{T}+N_{1}+N_{1}^{T} \text {, }
$$$$
\Xi_{55}=-Q_{2}-N_{2}-N_{2}^{T} \text {, }
$$$$
\Xi_{61}=\beta \Sigma+W_{1}^{T} P,
$$$$
\Psi_{22}=h_{M} R_{1}+d_{M} R_{2} \text {, }
$$$$
\Lambda=\left[\begin{array}{llllllllll}
-A & K & 0 & 0 & 0 & W_{1} & W_{2} & -I & K
\end{array}\right],
$$$$
\Gamma=\left[\begin{array}{lllllllll}
0 & \sqrt{\lambda \varepsilon} K & 0 & 0 & 0 & 0 & 0 & 0 & \sqrt{\lambda \varepsilon} K
\end{array}\right],
$$$$
\Pi_{1}=\left[\begin{array}{llllllllll}
d_{M} M_{1}^{T} & 0 & 0 & d_{M} M_{2}^{T} & 0 & 0 & 0 & 0 & 0
\end{array}\right] \text {, }
$$

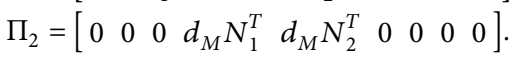

holds. 
Proof. Choosing an augmented LKF as follows:

where

$$
V(t, \eta(t))=\sum_{i=1}^{3} V_{i}(t, \eta(t))
$$

$$
\begin{aligned}
& V_{1}(t, \eta(t))=\eta^{T}(t) P \eta(t) \\
& V_{2}(t, \eta(t))=\int_{t-h_{M}}^{t} \eta^{T}(s) Q_{1} \eta(s) \mathrm{d} s+\int_{t-d_{M}}^{t} \eta^{T}(s) Q_{2} \eta(s) \mathrm{d} s \\
& V_{3}(t, \eta(t))=h_{M} \int_{t-h_{M}}^{t} \int_{\theta}^{t} \dot{\eta}^{T}(s) R_{1} \dot{\eta}(s) \mathrm{d} s \mathrm{~d} \theta+\int_{t-d_{M}}^{t} \int_{\theta}^{t} \dot{\eta}^{T}(s) R_{2} \dot{\eta}(s) \mathrm{d} s \mathrm{~d} \theta
\end{aligned}
$$

Calculating the derivative of $V(t, \eta(t))$ along the tra- where jectory of system (17):

$$
\dot{V}(t, \eta(t))=\sum_{i=1}^{3} \dot{V}_{i}(t, \eta(t))
$$

$$
\begin{aligned}
\dot{V}_{1}(t, \eta(t))= & 2 \eta^{T}(t) P\left[-A \eta(t)+W_{1} g(\eta(t))+W_{2} g(\eta(t-d(t)))+K\left(\eta(t-h(t))+e_{k}(t)\right)-\psi(u(t))\right], \\
\dot{V}_{2}(t, \eta(t))= & \eta^{T}(t)\left(Q_{1}+Q_{2}\right) \eta(t)-\eta^{T}\left(t-h_{M}\right) Q_{1} \eta\left(t-h_{M}\right) \\
& -\eta^{T}\left(t-d_{M}\right) Q_{2} \eta\left(t-d_{M}\right), \\
\dot{V}_{3}(t, \eta(t))= & \dot{\eta}^{T}(t)\left(h_{M}^{2} R_{1}+d_{M} R_{2}\right) \dot{\eta}(t)-h_{M} \int_{t-h_{M}}^{t} \dot{\eta}^{T}(s) R_{1} \dot{\eta}(s) \mathrm{d} s \\
& -\int_{t-d_{M}}^{t} \dot{\eta}^{T}(s) R_{2} \dot{\eta}(s) \mathrm{d} s .
\end{aligned}
$$

Thanks to Lemma 2, we know that if (22b) holds, we have

$$
-h_{M} \int_{t-h_{M}}^{t} \dot{\eta}^{T}(s) R_{1} \dot{\eta}(s) \mathrm{d} s \leq \xi_{1}^{T}(t) Q \xi_{1}(t),
$$

where

$$
\begin{aligned}
\mathcal{Q} & =\left[\begin{array}{ccc}
-R_{1} & * & * \\
R_{1}-U & -2 R_{1}+U+U^{T} & * \\
U & R_{1}-U & -R_{1}
\end{array}\right], \\
\xi_{1}(t) & =\left[\begin{array}{lll}
\eta^{T}(t) & \eta^{T}(t-h(t)) & \eta^{T}\left(t-h_{M}\right)
\end{array}\right]^{T} .
\end{aligned}
$$

Using the free-weight matrix (FWM) method, we can easily get that

$$
2 \xi^{T}(t) M\left[\eta(t)-\eta(t-d(t))-\int_{t-d(t)}^{t} \dot{\eta}(s) \mathrm{d} s\right]=0,
$$

$$
2 \xi^{T}(t) N\left[\eta(t-d(t))-\eta\left(t-d_{M}\right)-\int_{t-d_{M}}^{t-d(t)} \dot{\eta}(s) \mathrm{d} s\right]=0,
$$

where

$$
\begin{aligned}
& M=\left[\begin{array}{lllllllll}
M_{1}^{T} & 0 & 0 & M_{2}^{T} & 0 & 0 & 0 & 0 & 0
\end{array}\right]^{T}, \\
& N=\left[\begin{array}{lllllllll}
0 & 0 & 0 & N_{1}^{T} & N_{2}^{T} & 0 & 0 & 0 & 0
\end{array}\right]^{T}, \\
& \xi^{T}(t)=\left[\begin{array}{llllllll}
\eta^{T}(t) & \eta^{T}(t-h(t)) & \eta^{T}\left(t-h_{M}\right) & \eta^{T}(t-d(t)), \eta^{T}\left(t-d_{M}\right) & g^{T}(\eta(t)) & g^{T}(\eta(t-d(t))) & \psi(u(t))^{T} & e_{k}^{T}(t)
\end{array}\right],
\end{aligned}
$$


and note that

$$
\begin{aligned}
-2 \xi^{T}(t) M \int_{t-d(t)}^{t} \dot{\eta}(s) \mathrm{d} s \leq & d(t) \xi(t)^{T} M R_{2}^{-1} M^{T} \xi(t) \\
& +\int_{t-d(t)}^{t} \dot{\eta}^{T}(s) R_{2} \dot{\eta}(s) \mathrm{d} s \\
-2 \xi^{T}(t) N \int_{t-d_{M}}^{t-d(t)} \dot{\eta}(s) \mathrm{d} s \leq & \left(d_{M}-d(t)\right) \xi(t)^{T} N R_{2}^{-1} N^{T} \xi(t) \\
& +\int_{t-d_{M}}^{t-d(t)} \dot{\eta}^{T}(s) R_{2} \dot{\eta}(s) \mathrm{d} s .
\end{aligned}
$$

Combining (30) and (32), then we can easily get

$$
\begin{aligned}
-\int_{t-d_{M}}^{t} \dot{\eta}^{T}(s) R_{2} \dot{\eta}(s) \mathrm{d} s \leq & 2 \xi^{T}(t) M[\eta(t)-\eta(t-d(t))] \\
& +d(t) \xi(t)^{T} M R_{2}^{-1} M^{T} \xi(t) \\
& +2 \xi^{T}(t) N\left[\eta(t-d(t))-\eta\left(t-d_{M}\right)\right] \\
& +\left(d_{M}-d(t)\right) \xi(t)^{T} N R_{2}^{-1} N^{T} \xi(t) .
\end{aligned}
$$

Considering the inequality (13), obviously, we have

$$
g_{i}\left(\eta_{i}(t)\right)\left[g_{i}\left(\eta_{i}(t)\right)-\varrho_{i} \eta_{i}(t)\right] \leq 0,
$$

and here, we set $\Sigma=\operatorname{diag}\left\{\varrho_{1}, \varrho_{2}, \ldots, \varrho_{n}\right\}$; we can see that there exists a scalar $\alpha>0$ that makes the inequality

$$
-2 \alpha g^{T}(\eta(t)) g(\eta(t))+2 \alpha \Sigma g^{T}(\eta(t)) \eta(t) \geq 0,
$$

hold, and also, we know that there exists a scalar $\beta>0$ that makes the inequality

$$
-2 \beta g^{T}(\eta(t-d(t))) g(\eta(t-d(t)))+2 \beta \Sigma g^{T}(\eta(t-d(t))) \eta(t-d(t)) \geq 0,
$$

hold.

Combining inequalities (27), (28), and (33), considering ETS (9), saturation condition (16), and inequalities (35), (36), we can get

$$
\begin{aligned}
\dot{V}(t, \eta(t)) \leq & 2 \eta^{T}(t) P\left[-A \eta(t)+W_{1} g(\eta(t))+W_{2} g(\eta(t-d(t)))+K\left(\eta(t-h(t))+e_{k}(t)\right)-\psi(u(t))\right]+\xi_{1}^{T}(t) Q \xi_{1}(t) \\
& +\eta^{T}(t)\left(Q_{1}+Q_{2}\right) \eta(t)-\eta^{T}\left(t-h_{M}\right) Q_{1} \eta\left(t-h_{M}\right) \\
& -\eta^{T}\left(t-d_{M}\right) Q_{2} \eta\left(t-d_{M}\right)+\dot{\eta}^{T}(t)\left(h_{M}^{2} R_{1}+d_{M} R_{2}\right) \dot{\eta}(t) \\
& +d(t) \xi(t)^{T} M R_{2}^{-1} M^{T} \xi(t)+\left(d_{M}-d(t)\right) \xi(t)^{T} N R_{2}^{-1} N^{T} \xi(t) \\
& +2 \xi^{T}(t) M[\eta(t)-\eta(t-d(t))]+2 \xi^{T}(t) N\left[\eta(t-d(t))-\eta\left(t-d_{M}\right)\right] \\
& +\sigma(t) \eta^{T}(t-h(t)) \Omega \eta(t-h(t))-e_{k}^{T}(t) \Omega e_{k}(t) \\
& +\lambda \varepsilon\left(K \eta(t-h(t))+K e_{k}(t)\right)^{T}\left(K \eta(t-h(t))+K e_{k}(t)\right) \\
& -\lambda \psi(u(t))^{T} \psi(u(t))-2 \alpha g^{T}(\eta(t)) g(\eta(t))+2 \alpha \Sigma g^{T}(\eta(t)) \eta(t) \\
& -2 \beta g^{T}(\eta(t-d(t))) g(\eta(t-d(t)))+2 \beta \Sigma g^{T}(\eta(t-d(t))) \eta(t-d(t))
\end{aligned}
$$

Finally, based on Lemma 3 and the Schur complement, we can derive matrix inequalities (22a) from inequality (37), so if condition (22a) holds, we can ensure that synchronization error system (17) with actuator saturation is asymptotically stable. This completes the proof.

Remark 2. Consider the nonlinear terms $P K$ and $K^{T} P$ of matrix inequalities (22a) in Theorem 1 . In order to facilitate the following derivation, next, we will eliminate the nonlinear terms and design a synchronization controller in Theorem 2.

Theorem 2. For given parameters $h_{M}>0$ and $d_{M}>0$, scalars $\rho_{1}$ and $\rho_{2}$, and positive scalars $\lambda, \alpha, \beta$, and $\varepsilon, \sigma(0) \in(0,1)$, under AETS (9) and the feedback gain $K=P^{-1} Y$, synchronization error system (17) is asymptotically stable, if there 
exist appropriate matrices $P>0, Q_{i}>0, R_{i}>0, M_{i}, N_{i}(i=1,2)$ and $U$, which satisfy the following matrix inequalities:

$$
\begin{array}{r}
\widehat{\Psi}_{i}=\left[\begin{array}{cccc}
\widehat{\Xi} & * & * & * \\
\widehat{\Lambda} & \rho_{1}^{2} \Psi_{22}-2 \rho_{1} P & * & * \\
\widehat{\Gamma} & 0 & \rho_{2}^{2} I-2 \rho_{2} P & * \\
\Pi_{i} & 0 & 0 & -d_{M} R_{2}
\end{array}\right]<0 \quad(i=1,2), \\
{\left[\begin{array}{cc}
R_{1} & * \\
U & R_{1}
\end{array}\right]>0,}
\end{array}
$$

where

$$
\begin{aligned}
& \widehat{\Xi}=\left[\begin{array}{ccccccccc}
\Xi_{11} & * & * & * & * & * & * & * & * \\
\widehat{\Xi}_{21} & \Xi_{22} & * & * & * & * & * & * & * \\
U & R_{1}-U & \Xi_{33} & * & * & * & * & * & * \\
\Xi_{41} & 0 & 0 & \Xi_{44} & * & * & * & * & * \\
0 & 0 & 0 & -N_{1}^{T}+N_{2} & \Xi_{55} & * & * & * & * \\
\Xi_{61} & 0 & 0 & 0 & 0 & -2 \alpha I & * & * & * \\
W_{2}^{T} P & 0 & 0 & \beta \Sigma & 0 & 0 & -2 \beta I & * & * \\
-P & 0 & 0 & 0 & 0 & 0 & 0 & -\lambda I & * \\
Y^{T} & 0 & 0 & 0 & 0 & 0 & 0 & 0 & -\Omega
\end{array}\right] \text {, } \\
& \widehat{\Xi}_{21}=Y^{T}+R_{1}-U \text {, } \\
& \widehat{\Lambda}=\left[\begin{array}{lllllllll}
-P A & Y & 0 & 0 & 0 & P W_{1} & P W_{2} & -P & Y
\end{array}\right] \text {, } \\
& \widehat{\Gamma}=\left[\begin{array}{lllllllll}
0 & \sqrt{\lambda \varepsilon} Y & 0 & 0 & 0 & 0 & 0 & 0 & \sqrt{\lambda \varepsilon} Y
\end{array}\right] \text {, }
\end{aligned}
$$

and others are defined as in Theorem 1.

Proof. First, defining $\mathcal{J}=\operatorname{diag}\{\underbrace{I, I, \ldots I}_{99}, P, P, I\}$ and pre- and postmultiplying equation (22a) with $\mathscr{F}$; set the feedback gain $K=P^{-1} Y$, so we have $P K=Y$ and replace $P K$ with $Y$; then, we can get

$$
\widetilde{\Psi}_{i}=\left[\begin{array}{cccc}
\widehat{\Xi} & * & * & * \\
\widehat{\Lambda} & -P \Psi_{22}^{-1} P & * & * \\
\widehat{\Gamma} & 0 & -P I P & * \\
\Pi_{i} & 0 & 0 & -d_{M} R_{2}
\end{array}\right]<0 \quad(i=1,2)
$$

where $\widehat{\Xi}, \widehat{\Lambda}$ and $\widehat{\Gamma}$ are defined in (39).

Next, consider the Lemma 4, then we can get

$$
\begin{gathered}
-P \Psi_{22}^{-1} P \leq \rho_{1}^{2} \Psi_{22}-2 \rho_{1} P, \\
-P I P \leq \rho_{2}^{2} I-2 \rho_{2} P .
\end{gathered}
$$

Finally, combining (40) and (41), it is easy to get (38a). This completes the proof.

Remark 3. Considering that the nonlinear terms $-P \Psi_{22}^{-1} P$ and $-P I P$ appear when deriving Theorem 2. The corresponding matrix inequalities cannot be solved directly by the MATLAB LMI toolbox. And the cone complementarity linearization (CCL) method is more complex, so Lemma 4 with greatly reduced computational complexity is chosen here.

\section{Numerical Examples}

In this part, we will verify the feasibility of the proposed method through two numerical examples. By applying Theorem 2, some necessary parameters of the system are obtained, and numerical simulation results are given, which show the superiority of the proposed scheme. 
Example 1. According to Theorem 2 and considering the following parameters of system (1), as shown in [38],

$$
\begin{aligned}
A & =\left[\begin{array}{ll}
1.1 & 0 \\
0 & 1.9
\end{array}\right], \\
W_{1} & =\left[\begin{array}{cc}
-3.9+\frac{\pi}{4} & 5.1 \\
3.0 & -5.9+\frac{\pi}{4}
\end{array}\right], \\
W_{2} & =\left[\begin{array}{cc}
-0.2 \frac{\sqrt{2} \pi}{4} & -4.6 \\
1.9 & -3.2 \frac{\sqrt{2} \pi}{4}
\end{array}\right],
\end{aligned}
$$

and we choose the following activation functions:

$$
f_{i}(s)=\tanh (s), \quad(i=1,2) .
$$

Furthermore, it satisfies (4) with $\varrho_{i}=1,(i=1,2)$, so we have

$$
\Sigma=\operatorname{diag}\{1,1\} \text {. }
$$

Next, we set $\mu_{1}=\mu_{2}=3, \vartheta=9, \rho_{1}=\rho_{2}=1, \lambda=20$, $\varepsilon=0.2, d_{M}=0.2$, and $h_{M}=0.1$; by applying Theorem 2 with the adaptive threshold parameter $\sigma(0)=0.2$ and MATLAB LMI toolbox, we can obtain the following feasible solutions:

$$
\begin{aligned}
Q_{1} & =\left[\begin{array}{cc}
9.0944 & -8.9342 \\
-8.9342 & 9.6386
\end{array}\right], \\
Q_{2} & =\left[\begin{array}{cc}
4.4908 & -4.4239 \\
-4.4239 & 4.8030
\end{array}\right], \\
R_{1} & =\left[\begin{array}{cc}
7.0990 & -7.1390 \\
-7.1390 & 8.2316
\end{array}\right], \\
R_{2} & =\left[\begin{array}{cc}
11.7549 & -9.4840 \\
-9.4840 & 19.8103
\end{array}\right], \\
M_{1} & =\left[\begin{array}{cc}
-54.1427 & 32.6071 \\
32.4849 & -76.0327
\end{array}\right], \\
M_{2} & =\left[\begin{array}{cc}
45.5841 & -7.4072 \\
-23.2008 & 47.0170
\end{array}\right], \\
N_{1} & =\left[\begin{array}{cc}
-58.0500 & 47.0374 \\
47.0603 & -98.3077
\end{array}\right], \\
N_{2} & =\left[\begin{array}{cc}
8.3045 & -8.2542 \\
-8.2136 & 9.0107
\end{array}\right], \\
P & =\left[\begin{array}{cc}
4.2154 & -3.5926 \\
-3.5926 & 6.4925
\end{array}\right], \\
Y & =\left[\begin{array}{cc}
0.8382 & -0.6657 \\
-0.9721 & 1.0285
\end{array}\right], \\
U & =\left[\begin{array}{cc}
-2.7736 & 2.7168 \\
2.7244 & -2.9578
\end{array}\right],
\end{aligned}
$$

More, the controller gain matrix $K$ and the event-triggered parameter matrix $\Omega$ can be obtained as follows:

$$
\begin{aligned}
& K=P^{-1} Y=\left[\begin{array}{cc}
0.1348 & -0.0433 \\
-0.0751 & 0.1344
\end{array}\right], \\
& \Omega=\left[\begin{array}{cc}
30.4974 & -30.9639 \\
-30.9639 & 36.4758
\end{array}\right] .
\end{aligned}
$$

Here, we set $h=0.05$ as the sampling period and choose $x(0)=\left[\begin{array}{ll}2.2 & -2.5\end{array}\right]^{T}$ and $y(0)=\left[\begin{array}{ll}-2.4 & 2.6\end{array}\right]^{T}$. Then, as shown in Figures 2 and 3 , we get the state responses of the master-slave system with saturation control input, and the trajectories of the saturation control signals Sat $(u(t))$ and $u(t)$ are given in Figures 4 and 5 . Figure 6 shows the state trajectories of synchronization error system (17).

Figure 7 depicts the trajectory of the adaptive threshold parameter $\sigma(t)$ with the initial value of $\sigma(0)=0.2$. And the threshold converges to a constant when the system tends to be stable. Figures 8 and 9 show the event-triggered release instants and intervals under the AETS and the ETS, respectively. There are all only 63 measurement signals sent out to the controller out of a total of $10 / h=200$, which takes only $31.5 \%$ of the whole measurement signals being transmitted when the simulation time is $10 \mathrm{~s}$. However, in the case of AETS, the trigger frequency of the initial period is higher than that of ETS, which can shorten the dynamic process.

Example 2. According to Theorem 2 and considering system (1) with the following parameters, as shown in [1],

$$
\begin{aligned}
A & =\left[\begin{array}{lll}
1 & 0 & 0 \\
0 & 1 & 0 \\
0 & 0 & 1
\end{array}\right], \\
W_{1} & =\left[\begin{array}{ccc}
2 & -1 & 0 \\
1.7 & 1.71 & 1.1 \\
-2.5 & -2.9 & 0.56
\end{array}\right], \\
W_{2} & =\left[\begin{array}{ccc}
1.5 & -0.4 & 1 \\
0.2 & 1.4 & -0.3 \\
0.1 & 0.3 & -0.7
\end{array}\right],
\end{aligned}
$$

and we choose the following activation functions

$$
f_{i}(s)=\tanh (s), \quad i=1,2,3 .
$$

Furthermore, it satisfies (4) with $\varrho_{i}=1,(i=1,2,3)$, so we have

$$
\Sigma=\operatorname{diag}\{1,1,1\}
$$

Here, we set $\mu_{1}=\mu_{2}=\mu_{3}=0.06, \vartheta=15, \rho_{1}=\rho_{2}=1$, $\lambda=15, \varepsilon=0.2$, and $d_{M}=0.8$; by applying Theorem 2 with different event-triggered parameters $\sigma$, we can obtain the corresponding admissible upper bound $h_{M}$ as shown in Table 1. From Table 1, we can get that with the increase of the event-triggered parameters $\sigma$, the admissible upper bound of $h_{M}$ decreases.

Next, Example 2 will be discussed with the above parameters and $\sigma=0.2, h_{M}=0.0616$. Then the controller gain 


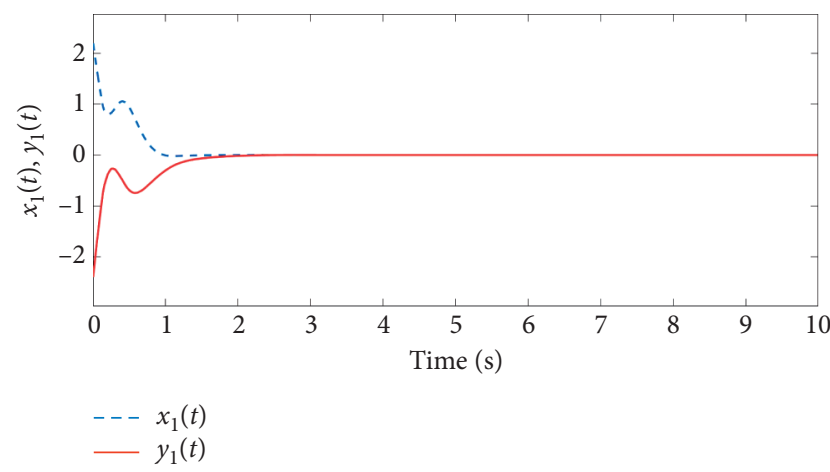

Figure 2: State responses of $x_{1}(t)$ and $y_{1}(t)$ under the controller in Example 1.

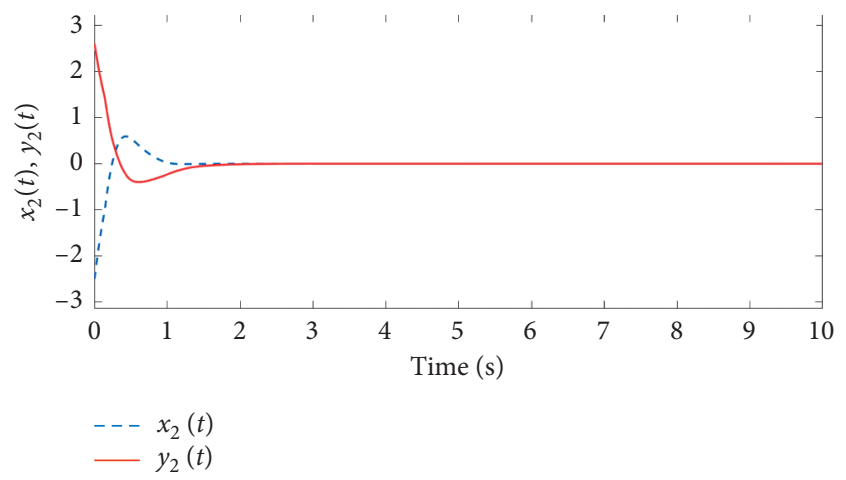

FIGURE 3: State responses of $x_{2}(t)$ and $y_{2}(t)$ under the controller in Example 1.

matrix $K$ and the event-triggered parameter matrix $\Omega$ can be obtained as follows:

$$
\begin{aligned}
K & =\left[\begin{array}{ccc}
-0.0945 & -0.0061 & -0.0380 \\
-0.0303 & -0.1584 & -0.0114 \\
-0.0071 & 0.0207 & 0.0200
\end{array}\right], \\
\Omega & =\left[\begin{array}{ccc}
0.2525 & 0.0039 & 0.1004 \\
0.0039 & 0.8307 & -0.2178 \\
0.1004 & -0.2178 & 0.7793
\end{array}\right] .
\end{aligned}
$$

The sampling period $h=0.05$ has to be set, and $x(0)=$ $\left[\begin{array}{lll}-1.5 & 2 & -2\end{array}\right]^{T}$ and $y(0)=\left[\begin{array}{lll}-2 & 1 & 0\end{array}\right]^{T}$ have to be chosen. The chaotic behaviors of the master-slave system are given in Figures 10(a) and 10(b). The state responses of master-slave system with saturation control input are shown in Figure $11\left(a_{1}\right)-\left(a_{3}\right)$. The trajectories of $u(t)$ are shown in Figure 12. Figure 13 shows the trajectories of the saturation control signals Sat $(u(t))$, and the state trajectories of synchronization error system (17) are shown in Figure 14.

Figure 15 depicts the trajectory of the adaptive threshold parameter $\sigma(t)$ with the initial value of $\sigma(0)=0.2$. Figure 16 illustrates the event-triggered release instants and intervals in Example 2, and there are 75 measurement signals sent to

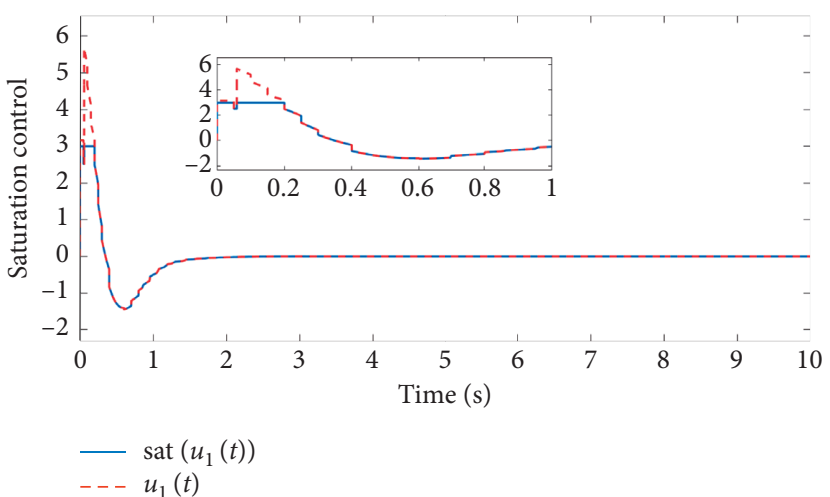

FIgURE 4: Trajectories of saturation control signal Sat $\left(u_{1}(t)\right)$ and $u_{1}(t)$ in Example 1.

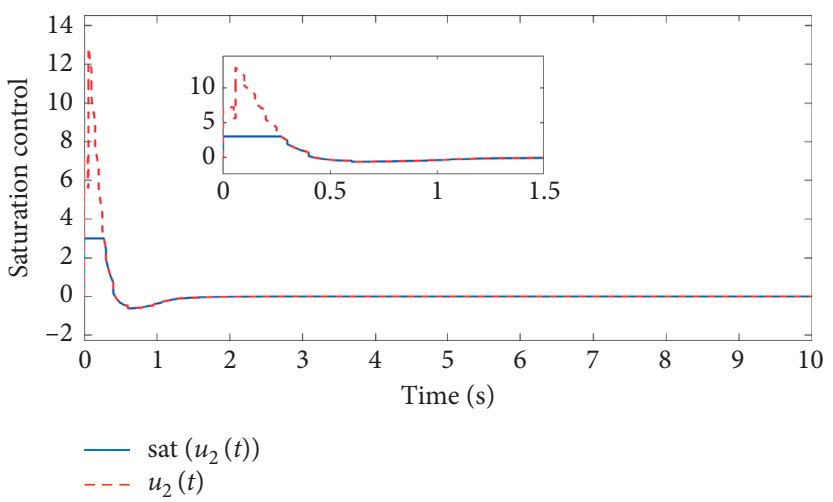

FIgURE 5: Trajectories of saturation control signal Sat $\left(u_{2}(t)\right)$ and $u_{2}(t)$ in Example 1.

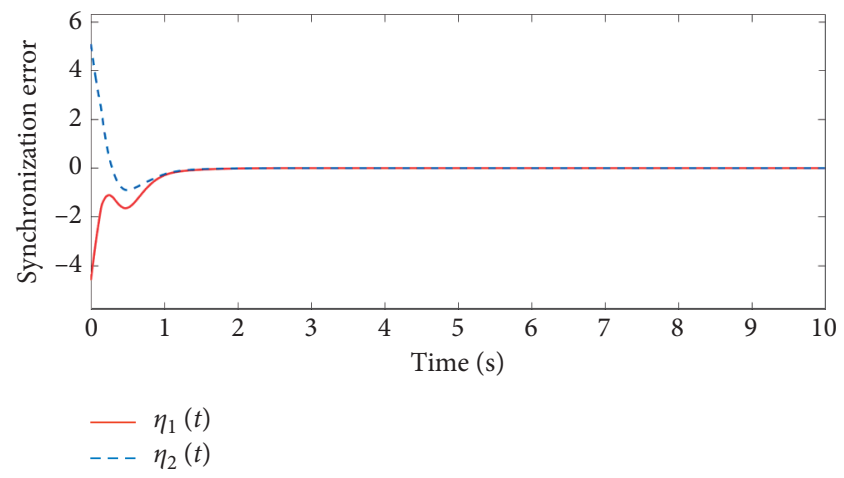

FIgURE 6: Trajectories of the synchronization error $\eta(t)$ in Example 1.

the controller out of a total of $25 / h=500$, which takes $15 \%$ of the whole measurement signals being transmitted when the simulation time is $25 \mathrm{~s}$. Simulation results show that the proposed method meets the expected requirements well, realizes the synchronization of the master-slave DNNs system, and effectively saves the limited network resources. 


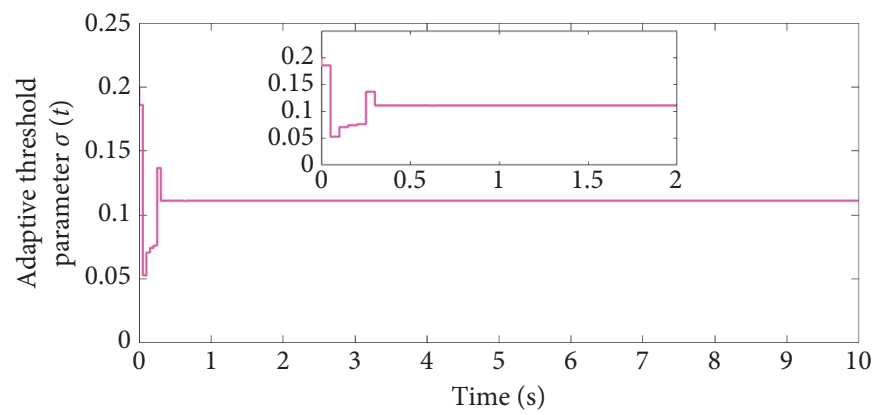

FIGURE 7: Trajectories of the adaptive threshold parameter $\sigma(t)$ in Example 1.

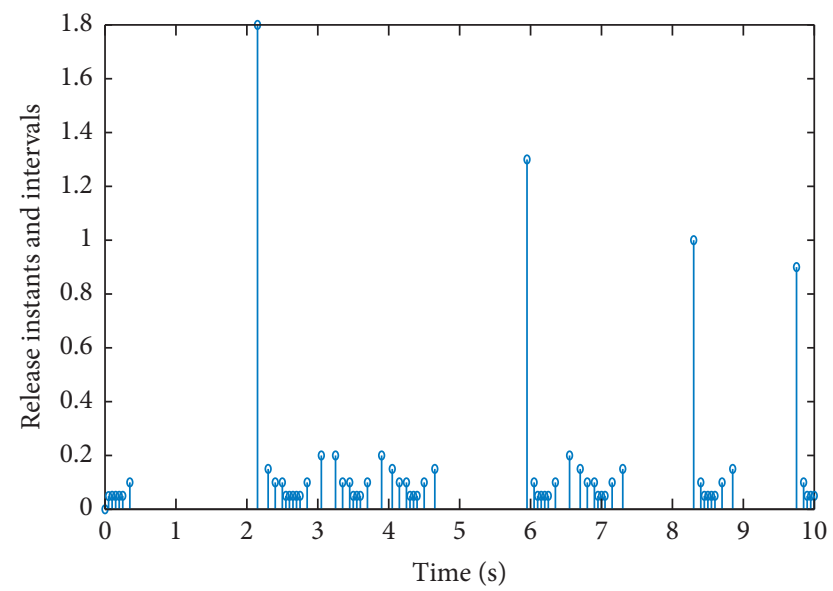

Figure 8: The release instants and intervals under the AETS in Example 1.

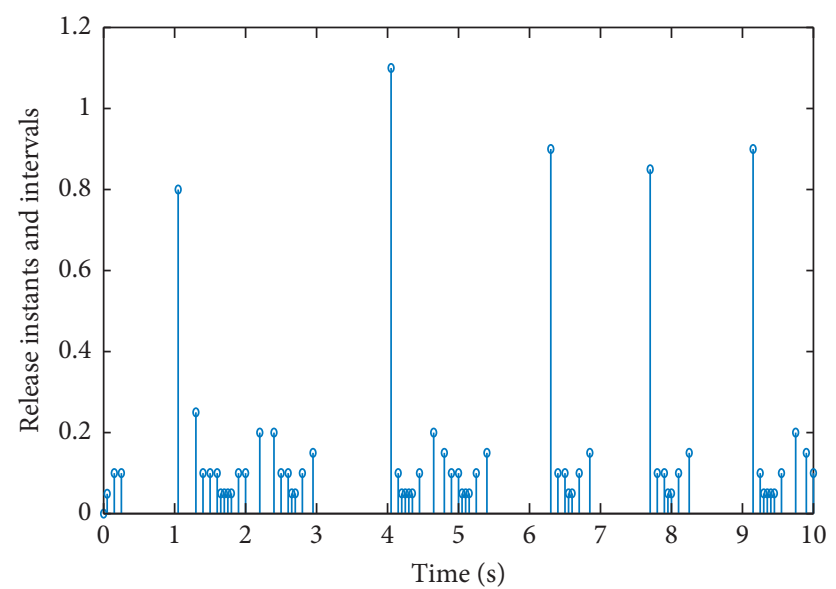

FIgURE 9: The release instants and intervals under the ETS in Example 1.

TABLE 1: Admissible upper bound $h_{M}$ for different event-triggered parameters $\sigma$.

\begin{tabular}{cccccccc}
\hline$\sigma$ & 0.01 & 0.03 & 0.05 & 0.08 & 0.1 & 0.15 & 0.2 \\
\hline$h_{M}$ & 0.2428 & 0.2002 & 0.1718 & 0.1396 & 0.1222 & 0.0877 & 0.0616 \\
\hline
\end{tabular}




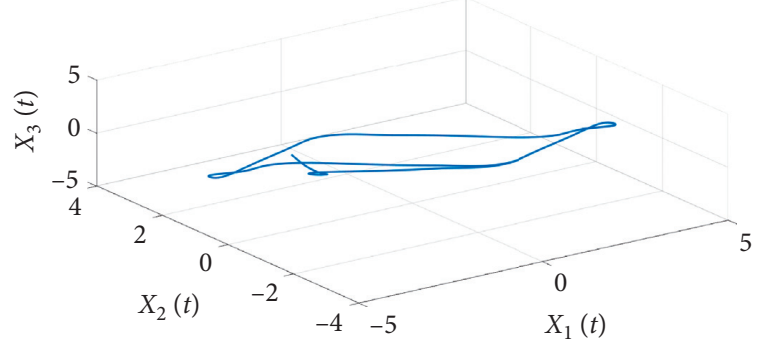

(a)

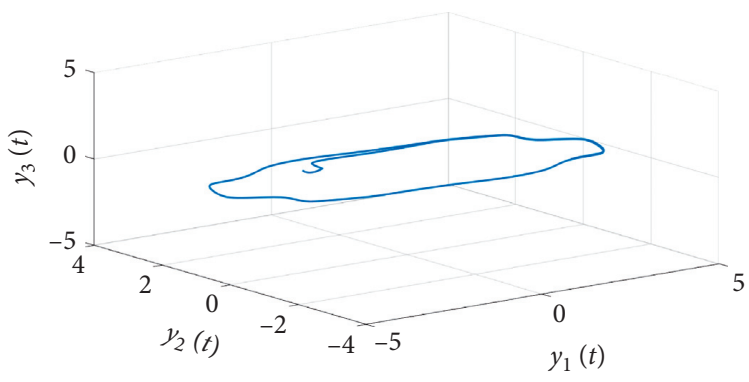

(b)

FIgURE 10: Chaotic behavior of the master system and slave system in Example 2.

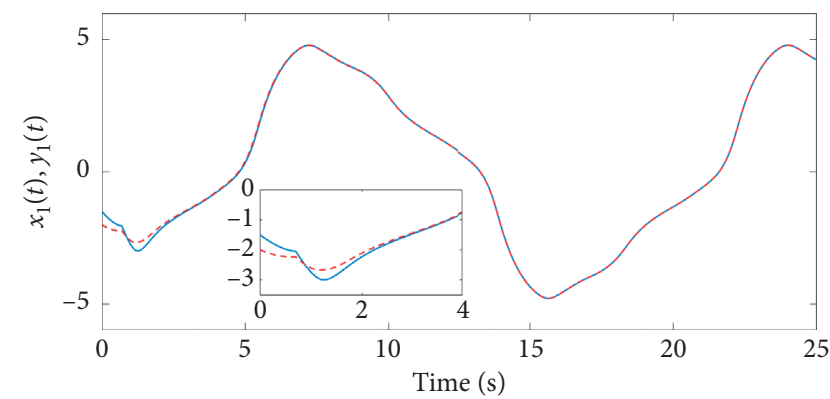

$x_{1}(t)$

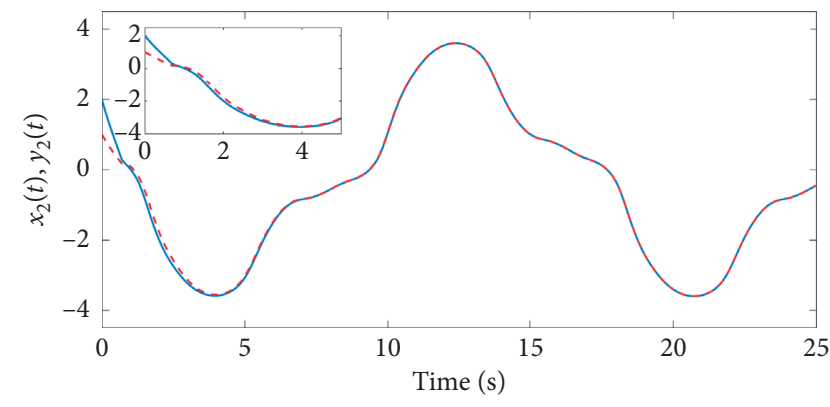

$-x_{2}(t)$

(a)

(b)

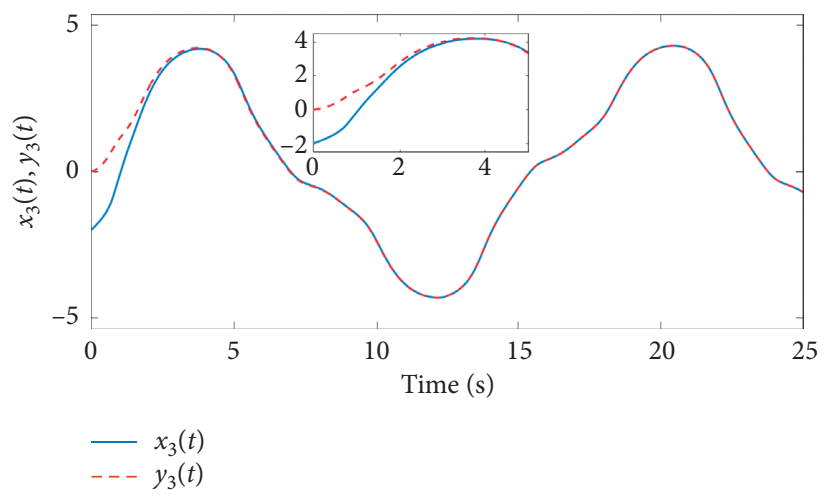

(c)

FIgURE 11: Trajectories of $x(t)$ and $y(t)$ in Example 2. 


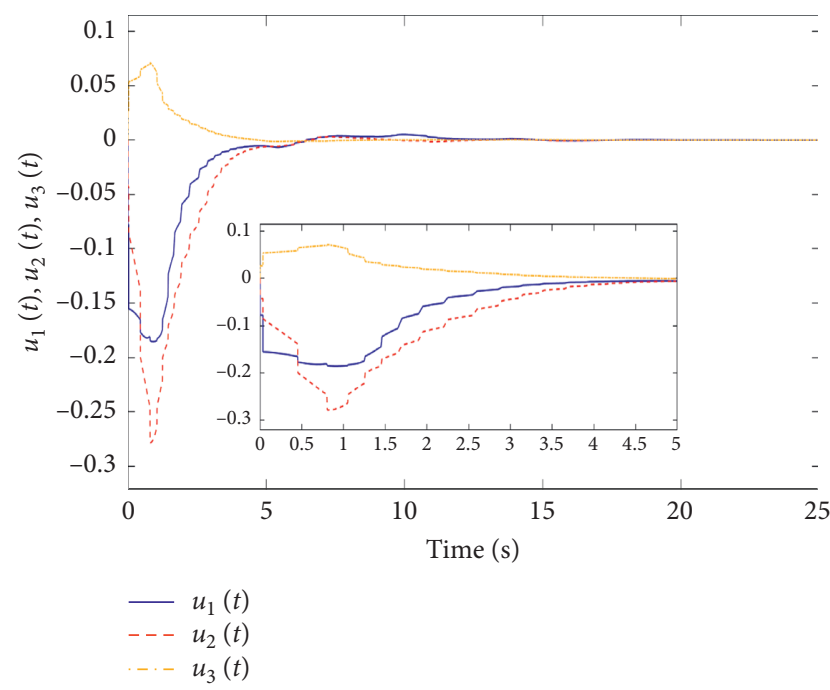

Figure 12: Trajectories of $u(t)$ in Example 2.

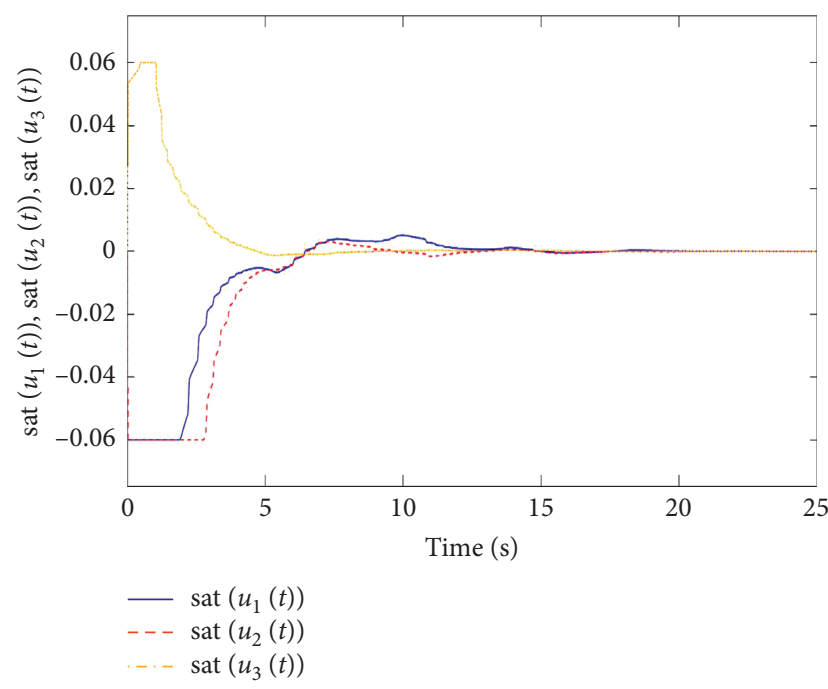

Figure 13: Trajectories of Sat $(u(t))$ in Example 2.

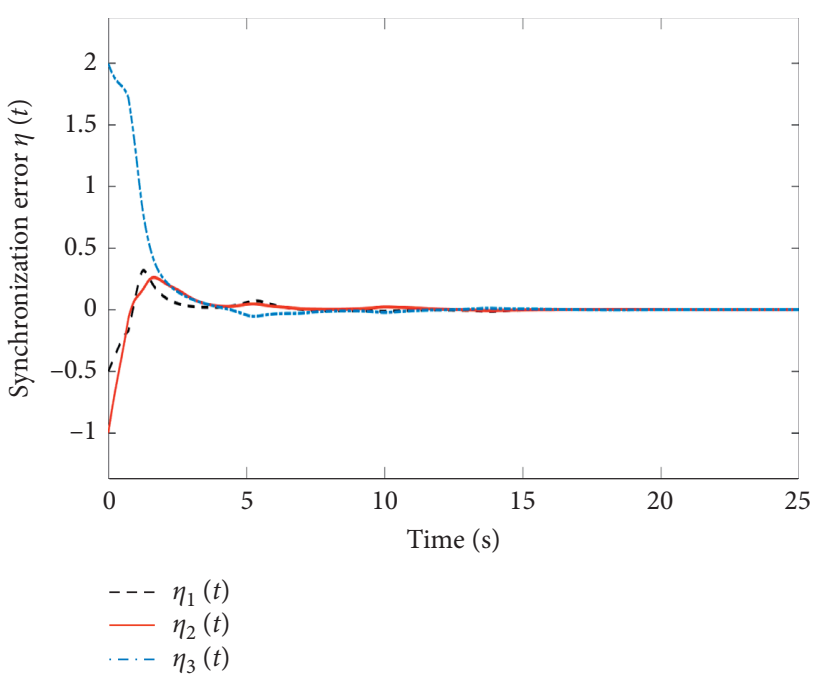

FIgURE 14: Trajectories of synchronization error $\eta(t)$ in Example 2.

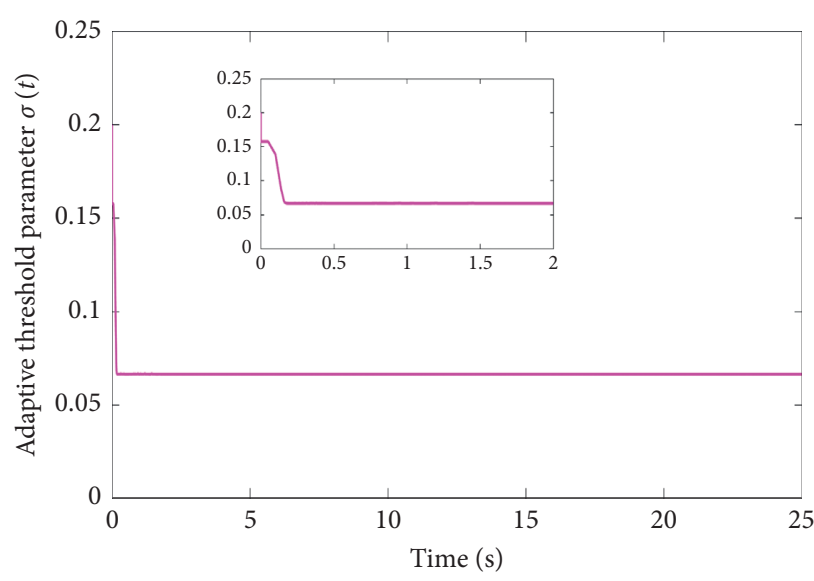

Figure 15: Trajectory of the adaptive threshold parameter $\sigma(t)$ in Example 2.

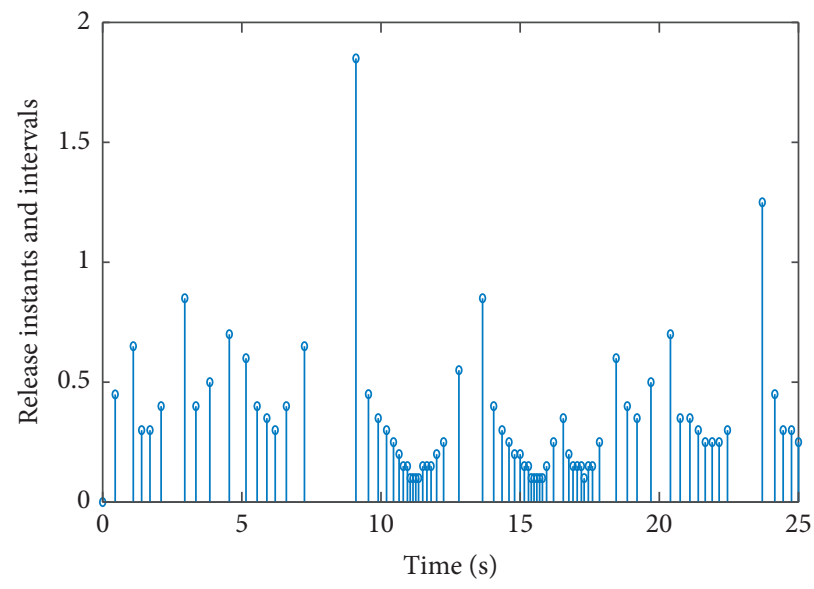

FIGURE 16: The event-triggered release instants and intervals in Example 2.

\section{Conclusion}

This paper discussed the event-triggered synchronization control problem for DNNs with actuator saturation. Considering the limited communication channel capacity of the network system, we introduced AETS. Under the AETS, we transformed the synchronization problem of master-slave DNNs into the stability problem of the synchronization error system, and the error system model is constructed with actuator saturation based on an NCS framework. By employing Lyapunov stability theory, a new sufficient criterion to guarantee the asymptotic stability of the synchronization error system is obtained; then, we got some parameters related to controller and event trigger by solving LMIs. Lastly, we give two numerical examples to prove the effectiveness of the proposed method; simulation results show that this method meets the expected requirement well, realizes the synchronization of the master-slave DNNs, and saves the limited network resources effectively. Considering that there are many unpredictable factors in the real operating environment, such as strong signal attenuation and cyberattacks [39], the synchronization and state estimation 
problem of the switched neural network based on ETS under the cyberattacks will be further studied in the future $[40,41]$.

\section{Data Availability}

The data used to support the findings of this study are included within the article.

\section{Conflicts of Interest}

The authors declare that they have no conflicts of interest.

\section{Acknowledgments}

This work was supported by the National Natural Science Foundation of China under grant no. 61573095.

\section{References}

[1] L. Li, W. Zou, and S. Fei, "Event-triggered synchronization of delayed neural networks with actuator saturation using quantized measurements," Journal of the Franklin Institute, vol. 356, no. 12, pp. 6433-6459, 2019.

[2] L. Sun, Y. Tang, W. Wang, and S. Shen, "Stability analysis of time-varying delay neural networks based on new integral inequalities," Journal of the Franklin Institute, vol. 357, no. 15, pp. 10828-10843, 2020.

[3] H. Liang, L. Zhang, Y. Sun, and T. Huang, "Containment control of semi-Markovian multiagent systems with switching topologies," IEEE Transactions on Systems, Man, and Cybernetics: Systems, vol. 51, no. 6, pp. 3889-3899, 2021.

[4] X.-M. Zhang and Q.-L. Han, "Global asymptotic stability for a class of generalized neural networks with interval timevarying delays," IEEE Transactions on Neural Networks, vol. 22, no. 8, pp. 1180-1192, 2011.

[5] J. Cao, G. Chen, and P. Li, "Global synchronization in an array of delayed neural networks with hybrid coupling," IEEE Transactions on Systems, Man, and Cybernetics, Part B (Cybernetics), vol. 38, no. 2, pp. 488-498, 2008.

[6] X. Li, D. O'Regan, and H. Akca, "Global exponential stabilization of impulsive neural networks with unbounded continuously distributed delays," IMA Journal of Applied Mathematics, vol. 80, no. 1, pp. 85-99, 2015.

[7] X. Lv, X. Li, J. Cao, and P. Duan, "Exponential synchronization of neural networks via feedback control in complex environment," Complexity, vol. 2018, Article ID 4352714, 13 pages, 2018.

[8] R. Rakkiyappan, S. Dharani, and J. Cao, "Synchronization of neural networks with control packet loss and time-varying delay via stochastic sampled-data controller," IEEE Transactions on Neural Networks and Learning Systems, vol. 26, no. 12, pp. 3215-3226, 2015.

[9] G. Chen, J. Sun, and J. Xia, "Estimation of domain of attraction for aperiodic sampled-data switched delayed neural networks subject to actuator saturation," IEEE Transactions on Neural Networks and Learning Systems, vol. 99, pp. 1-15, 2019.

[10] Y. Tan, Q. Liu, J. Liu, X. Xie, and S. Fei, “Observer-based security control for interconnected semi-Markovian jump systems with unknown transition probabilities," IEEE Transactions on Cybernetics, pp. 1-13, 2021.

[11] Y. Tan, M. Xiong, B. Zhang, and S. Fei, "Adaptive eventtriggered nonfragile state estimation for fractional-order complex networked systems with cyber attacks," IEEE Transactions on Systems, Man, and Cybernetics: Systems, pp. 1-13, 2021.

[12] Y. Wang, H. R. Karimi, and H. Yan, "Notice of violation of IEEE publication principles: an adaptive event-triggered synchronization approach for chaotic Lur'e systems subject to aperiodic sampled data," IEEE Transactions on Circuits and Systems II: Express Briefs, vol. 66, no. 3, pp. 442-446, 2019.

[13] H. Lu, Y. Deng, and W. Zhou, "Adaptive event-triggered Ho control for networked control systems with actuator saturation and random nonlinearities," IEEE Access, vol. 8, pp. 220723-220733, 2020.

[14] Y. Wang, H. Shen, and D. Duan, "On Stabilization of quantized sampled-data neural-network-based control systems," IEEE Transactions on Cybernetics, vol. 47, no. 10, pp. 3124-3135, 2017.

[15] Y.-G. Chen, Z.-D. Wang, B. Shen, and H.-L. Dong, "Exponential synchronization and L2-gain analysis of delayed chaotic neural networks via intermittent control with actuator saturation," IEEE Transactions on Neural Networks \& Learning Systems, vol. 30, no. 12, pp. 3722-3734, 2019.

[16] W. Qi, G. Zong, and H. R. Karimi, "Sliding mode control for nonlinear stochastic singular semi-Markov jump systems," IEEE Transactions on Automatic Control, vol. 65, no. 99, pp. 361-368, 2019.

[17] Z.-G. Wu, P. Shi, H. Su, and J. Chu, "Local synchronization of chaotic neural networks with sampled-data and saturating actuators," IEEE Transactions on Cybernetics, vol. 44, no. 12, pp. 2635-2645, 2014.

[18] F. Yang and Y. Li, "Set-membership filtering for systems with sensor saturation," Automatica, vol. 45, no. 8, pp. 1896-1902, 2009.

[19] M. Rehan, M. Tufail, and K.-S. Hong, "Delay-range-dependent synchronization of drive and response systems under input delay and saturation," Chaos, Solitons \& Fractals, vol. 87, pp. 197-207, 2016.

[20] Y. Tan, Y. Liu, B. Niu, and S. Fei, "Event-triggered synchronization control for T-S fuzzy neural networked systems with time delay," Journal of the Franklin Institute, vol. 357, no. 10, pp. 5934-5953, 2020.

[21] Z.-D. Wang, B. Shen, and X. Liu, "Ho filtering with randomly occurring sensor saturations and missing measurements," Automatica, vol. 48, no. 3, pp. 556-562, 2012.

[22] L. Sun, Y. Wang, and G. Feng, "Control design for a class of affine nonlinear descriptor systems with actuator saturation," IEEE Transactions on Automatic Control, vol. 60, no. 8, pp. 2195-2200, 2015.

[23] Y. Chen, Z. Wang, B. Shen, and H. Dong, "Exponential synchronization for delayed dynamical networks via intermittent control: dealing with actuator saturations," IEEE Transactions on Neural Networks and Learning Systems, vol. 30, no. 4, pp. 1000-1012, 2019.

[24] Z. Fei, C. Guan, and H. Gao, "Exponential synchronization of networked chaotic delayed neural network by a hybrid event trigger scheme," IEEE Transactions on Neural Networks \& Learning Systems, vol. 29, no. 99, pp. 2558-2567, 2018.

[25] X. Zhao, H. Yang, H.-R. Karimi, and Y. Zhu, "Adaptive neural control of MIMO nonstrict-feedback nonlinear systems with time delay," IEEE Transactions on Cybernetics, vol. 46, no. 6, pp. 1337-1349, 2016.

[26] Z. Li, F. Alsaadi, T. Hayat, and H. Gao, "New results on stability analysis and stabilisation of networked control system," IET Control Theory \& Applications, vol. 8, no. 16, pp. 1707-1715, 2014. 
[27] Y. Shi, J. Huang, and B. Yu, "Robust tracking control of networked control systems: application to a networked DC motor," IEEE Transactions on Industrial Electronics, vol. 60, no. 12, pp. 5864-5874, 2013.

[28] J. P. Hespanha, P. Naghshtabrizi, and Y. Xu, "A survey of recent results in networked control systems," Proceedings of the $45^{\text {th }}$ IEEE International Conference on Decision and Control, vol. 95, no. 1, pp. 138-162, 2007.

[29] S. Rajavel, R. Samidurai, J. Cao, A. Alsaedi, and B. Ahmad, "Finite-time non-fragile passivity control for neural networks with time-varying delay," Applied Mathematics and Computation, vol. 297, pp. 145-158, 2017.

[30] F. Gouaisbaut and D. Peaucelle, "Delay-dependent stability analysis of linear time delay systems," IFAC Proceedings Volumes, vol. 39, no. 10, pp. 54-59, 2006.

[31] H.-B. Zeng, K. L. Teo, and Y. He, "A new looped-functional for stability analysis of sampled-data systems," Automatica, vol. 82, pp. 328-331, 2017.

[32] H.-B. Zeng, Z.-L. Zhai, Y. He, K.-L. Teo, and W. Wang, "New insights on stability of sampled-data systems with time-delay," Applied Mathematics and Computation, vol. 374, Article ID 125041, 2020.

[33] K. Liu, A. Seuret, and Y. Xia, "Stability analysis of systems with time-varying delays via the second-order Bessel-Legendre inequality," Automatica, vol. 76, pp. 138-142, 2017.

[34] Y.-B. Huang, Y. He, J. An, and M. Wu, "Polynomial-type Lyapunov-Krasovskii functional and Jacobi-Bessel inequality: further results on stability analysis of time-delay systems," IEEE Transactions on Automatic Control, vol. 66, no. 6, pp. 2905-2912, 2021.

[35] C.-K. Zhang, F. Long, Y. He, W. Yao, L. Jiang, and M. Wu, “A relaxed quadratic function negative-determination lemma and its application to time-delay systems," Automatica, vol. 113, Article ID 108764, 2020.

[36] E. Tian, D. Yue, and Y. Zhang, "Delay-dependent robust $H \infty$ control for T-S fuzzy system with interval time-varying delay," Fuzzy Sets and Systems, vol. 160, no. 12, pp. 1708-1719, 2009.

[37] C. Peng, Q.-L. Han, and D. Yue, “To transmit or not to transmit: a discrete event-triggered communication scheme for networked Takagi-Sugeno fuzzy systems," IEEE Transactions on Fuzzy Systems, vol. 21, no. 1, pp. 164-170, 2013.

[38] C. Pradeep, Y. Cao, R. Murugesu, and R. Rakkiyappan, “An event-triggered synchronization of semi-Markov jump neural networks with time-varying delays based on generalized freeweighting-matrix approach," Mathematics and Computers in Simulation, vol. 155, pp. 41-56, 2019.

[39] J. Mao, Y. Sun, X. Yi, H. Liu, and D. Ding, "Recursive filtering of networked nonlinear systems: a survey," International Journal of Systems Science, vol. 52, no. 6, pp. 1110-1128, 2021.

[40] D. Ding, Q.-L. Han, X. Ge, and J. Wang, "Secure state estimation and control of cyber-physical systems: a survey," IEEE Transactions on Systems, Man, and Cybernetics: Systems, vol. 51, no. 1, pp. 176-190, 2021.

[41] G. Zong, H. Ren, and H. R. Karimi, "Event-triggered communication and annular finite-time $H \infty$ filtering for networked switched systems," IEEE Transactions on Cybernetics, vol. 51, no. 1, pp. 309-317, 2021. 